\title{
Avoiding Charge Induced Drift In Vitrified Biological Specimens Through Scanning Transmission Electron Microscopy
}

\author{
G. Nicotra, ${ }^{*}$ Hui-Ting Chou, ${ }^{* *}$ Henning Stahlberg,${ }^{* *}$ and N.D. Browning ${ }^{* * *}$
}

* Department of Physics \& Astronomy University of Catania Via S. Sofia 64, I-95123 Catania, Italy and CNR-IMM of Catania Stradale Primosole 50 I-95121 Catania, Italy

** Molecular \& Cellular Biology, Briggs Hall 5, University of California at Davis, 1 Shields Ave., Davis, CA 95616, USA

*** Department of Chemical Engineering and Materials Science University of California-Davis One Shields Ave Davis, CA 95616, and Materials Science Directorate, Lawrence Livermore National Laboratory P.O. Box 808; L-356 Livermore, CA 94550

Bright-field TEM imaging of vitrified biological samples traditionally suffers from image blurring due of the build-up of positive charge in the irradiated area (the point from which the secondary electrons are emitted) [1]. When specimens are tilted to high angles, the charging leads to a severe degradation in image resolution in the direction perpendicular to the tilt axis. One way to avoid such charging effects may be through the use of Scanning Transmission Electron Microscopy (STEM). STEM quantifies the amount of electrons that are scattered under a specific angle, but is largely insensitive to charge-induced lateral displacement of electrons during specimen transmittance. In the STEM mode of operating the microscope, a sub-nanometer electron beam scans the specimen, with the final image being acquired serially as a function of probe position in either a small on-axis bright field detector (equivalent to TEM imaging) or by a High Angle Annular Dark Field detector (HAADF). This HAADF technique is called Z-contrast STEM, and has been shown to provide an incoherent image at atomic resolution in crystalline materials where the interatomic spacing is larger than the probe size [2]. The small size of the beam and its stability allow us to obtain high resolution images [3], a factor of two higher than in a coherent image, and since the intensity directly reflects the mean square atomic number $(\mathrm{Z})$, information on the local atomic structure, chemical composition, and bonding at interfaces on the fundamental atomic scale can be obtained [4].

In this experiment, the STEM techniques were applied to vitrified Tobacco Mosaic Virus (TMV) with a diameter of $1.8 \mathrm{~nm}$ and the length around $100 \sim 300 \mathrm{~nm}$ using a JEOL 2100 STEM equipped with a FEG filament and operated at 200keV. TMV was vitrified in non-supported ice-layers spanning the holes of a perforated carbon film (Quantifoil) by quick-freezing in ethane slush and mounted in Gatan cryo holder at liquid nitrogen temperature for observation in the microscope. For the STEM analysis, a $0.5 \mathrm{~nm}$ of probe size, $3 \%$ reduced emission current, $40 \mu \mathrm{m}$ condenser aperture, and the HAADF detector with a camera length of $25 \mathrm{~mm}$ were used. The specimen was tilted at $45^{\circ}$, where TEM Bright Field images showed strong drift due to charging effects. In Fig.1(a), there is no clear evidence of charge-induced drift effect even if the TMV is difficult to observe in the first beam scan due to the ice thickness. An improvement is visible after the second scan, see Fig 1(b), due to partial ice melting, but the excess of beam dose damages the virus. In order to reduce the beam damage on the virus, the condenser aperture was reduced to $10 \mu \mathrm{m}$ (Fig. 2 (a) and (b)). However, under these operating conditions, the contrast appears too low to keep the virus visible with respect to the ice. Improving the experimental conditions (camera length and dose) should improve these results further and improve contrast. The two STEM images show a crystal of ice lying on the ice 
layer, obtained by tilting the specimen to $0^{\circ}$ and $45^{\circ}$. Both pictures were acquired using the smallest condenser aperture of $1 \mu \mathrm{m}$, in order to reduce ice melting, and a longer camera length of $50 \mathrm{~mm}$, in order to improve the signal. What we observe is that even at $45^{\circ}$ there is no dramatic resolution degradation effect due to electron charging-induced drift, since the crystal appears well in focus. This result represents the first clear evidence that the STEM approach could be a useful technique for the analysis of biological samples.

\section{References}

[1] R. Henderson, Ultramicroscopy 46, (1992) 1

[2] S. J. Pennycook, Ultramicroscopy 30 (1989) 58

[3] E.M. James, N.D. Browning, Ultramicroscopy 78 (1999) 125

[4] M.M.McGibbon et al, Science, 266 (1994) 102

[5] This research was supported by the US DOE under grant number DE-FG02-03ER46057, and by the NIGMS under grant number 1-U54-GM074929-01.
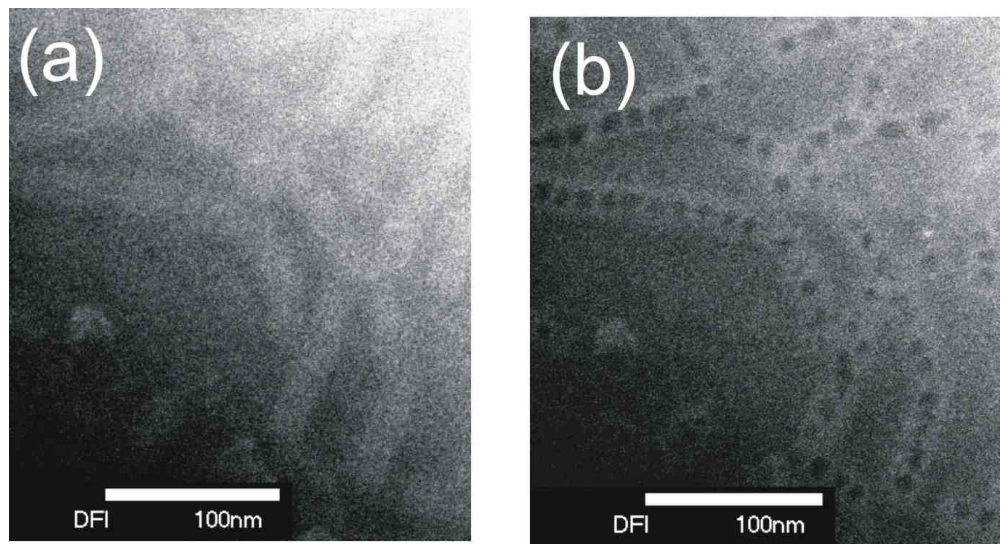

FIG. 1. Dark Field STEM Image of the TMV acquired at $45^{\circ}$ and low emission current and $3 \mu \mathrm{m}$ condenser aperture. a) after the first beam scan b) after the second beam scan. Although the images show no evidence of charge-induced drift, the beam dose is still high enough to cause damage.
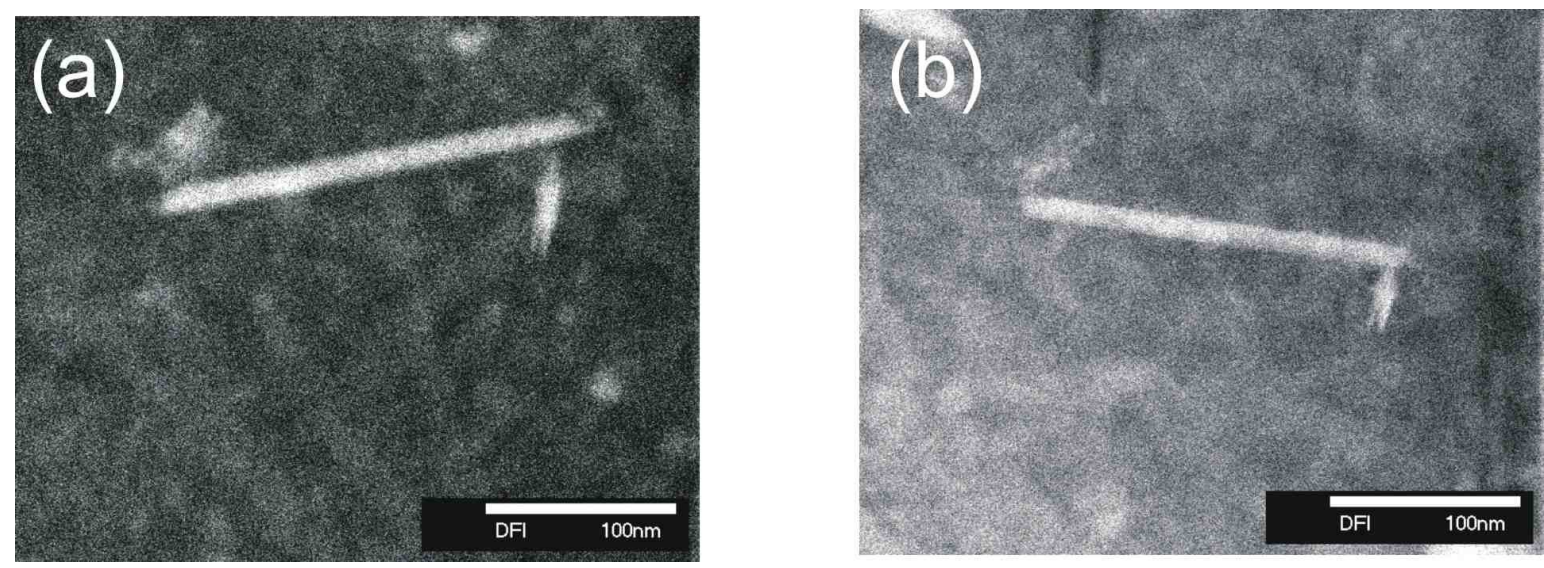

FIG. 2. Dark Field STEM Images of an ice crystal lying on ice layer. Images acquired at low emission current and $1 \mu \mathrm{m}$ condenser aperture a) no tilted specimen b) specimen tilted at $45^{\circ}$. Pictures show no evidence of charge-induced drift. 